DOI: $10.1515 / \mathrm{adms}-2017-0014$

\title{
Z. Usarek ${ }^{1 *}$, K. Warnke ${ }^{2}$
}

${ }^{1}$ Gdańsk University of Technology, Faculty of Applied Physics and Mathematics, Department of Solid State Physics, Gdańsk, Poland

${ }^{2}$ CDRiA Pipeline Services Ltd.

*zbigniew.usarek@pg.edu.pl

\section{INSPECTION OF GAS PIPELINES USING MAGNETIC FLUX LEAKAGE TECHNOLOGY}

\begin{abstract}
Magnetic non-destructive testing methods can be classified into the earliest methods developed for assessment of steel constructions. One of them is the magnetic flux leakage technology. A measurement of the magnetic flux leakage is quite commonly used for examination of large objects such as tanks and pipelines. Construction of a magnetic flux leakage tool is relatively simple, but a quantitative analysis of recorded data is a difficult task. Therefore, methods of magnetic flux leakage signal processing and analysis are still under development. A magnetic flux leakage in-line-inspection tool called FLUMAG 500 was constructed. FLUMAG 500 was designed for gas and oil pipelines inspection. In this paper principle of operation of FLUMAG 500 was described. Advanced algorithms of the signal processing and analysis was also developed. Results coming from the development stage as well as from the final construction of the tool were presented. Analysis of these results shows that FLUMAG 500 is a suitable tool for detection of corrosion defects in a pipeline wall.
\end{abstract}

Keywords: in -line inspection, magnetic flux leakage, pipeline inspection gauge

\section{INTRODUCTION}

Reliable operation of a transmission pipeline requires regular inspections of its technical condition to provide safe exploitation of such pipeline for a long time span. Corrosion is one of the most common factors leading to pipeline failures. It occurs on internal as well as on external surfaces of a pipe wall as demonstrated in Fig. 1. In addition to direct losses (cost of repairing or replacement of a failed construction, disruption of pipeline operation, etc.), also indirect losses should be taken into account, e.g. threads for public safety. Corrosion-related disasters are also dangerous for the environment, e.g. leakage of hazardous media into water and soil. In the case of a pipeline there are several methods of corrosion protection [1]: cathodic protection, corrosion control by inhibition, and protective coatings. However, regardless of the use of corrosion protection, it is necessary to constantly monitor pipeline technical condition. 
Significant number of operating pipelines are underground or laid on the seabed which makes direct access to the external surface of a pipe wall very difficult. Thus, the most popular methods of pipeline investigation rely on measurements performed inside a pipe. Such measurements are most often made by an inspection tool called 'pig'. Term 'pig' is often referred to as acronym for 'pipeline inspection gauge.' A pig can be designed to record various data such as: distance traveled by the pig, video data, internal geometry of a pipe, its wall thickness, curvature, temperature and pressure of transported medium, etc. There are two ways of pipeline examination:

- on-line. Measurements are done in real time. A tool operator receives data from an inspection tool through a cable. The inspection tool is often self-driven. This method is mainly used for testing short sections of plumbing, sewerage or temporary out of service pipelines;

- in-line. In this case a diagnostic tool has the piston-like form and moves inside an examined pipeline driven by the force of an extruded medium. Measurement data are collected and stored on-the-fly while the tool is moving. Obtained data are analyzed after the tool is removed from the pipeline.

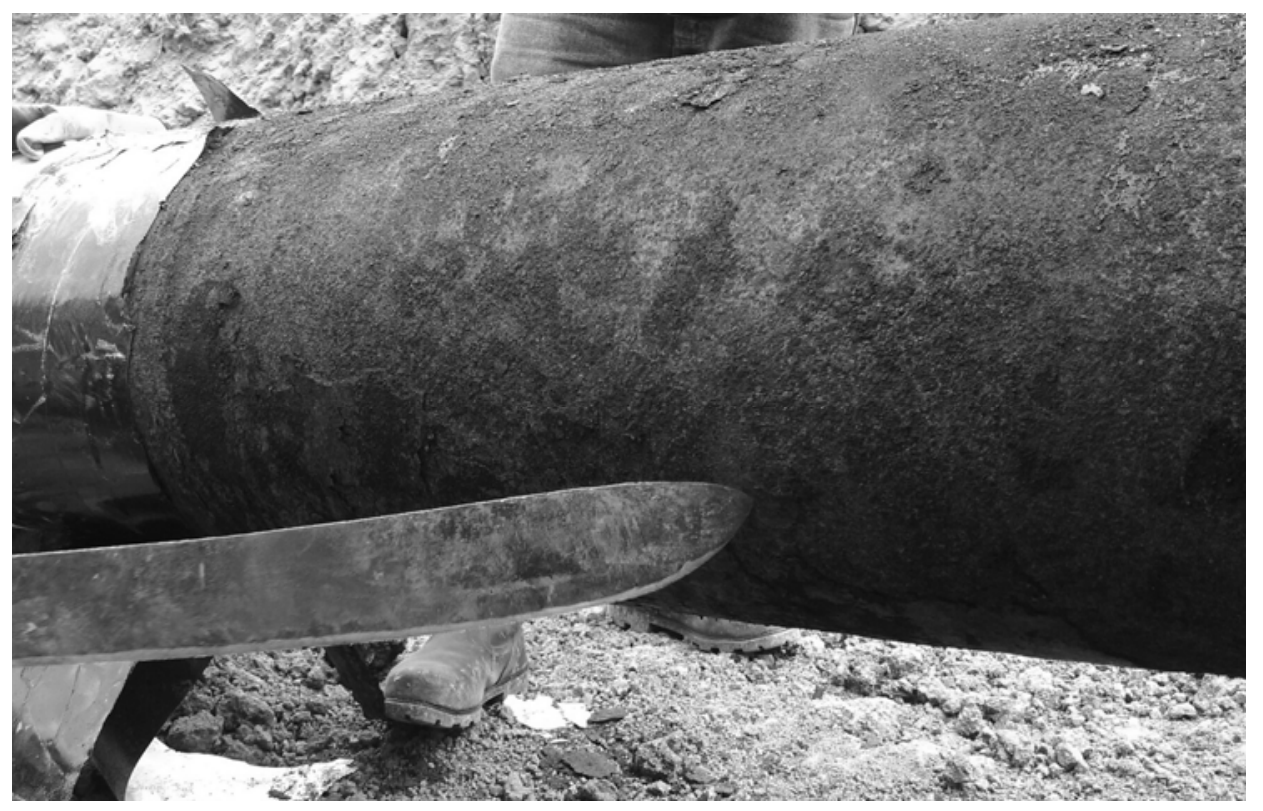

Fig. 1. Corroded pipe

The most relevant information about technical condition of a pipeline is obtained throughout measurements of pipe wall properties. Such measurements can be done in two ways: directly or indirectly. Direct methods include measurements of wall thickness using ultrasonic thickness gauges. In this method reduction of wall thickness referred to a nominal value may indicate a corrosion process occurring at a given measurement point. An in-line inspection pig called FLUMAG 500, designed in collaboration between CDRiA Ltd. and Gdansk University of Technology, uses an indirect method - magnetic flux leakage (MFL) technology - to perform measurements of pipeline technical condition. MFL technology is a non-destructive testing method commonly used to detect metal losses caused by corrosion in steel constructions [2]. This method is particularly useful for natural gas pipelines testing when ultrasonic testing (UT) generally can not be used because UT requires introducing of a liquid into the pipeline to couple the UT probe to the pipe wall $[3,4]$. 
MFL method involves the use of a source of strong magnetic flux, such as neodymium magnets, for magnetizing the pipe wall. The magnetic flux leaks from the wall in the location of a metal loss. Thus, the metal loss can be detected by a pig equipped with magnetic field sensors. Most pigs measure one or more components of magnetic flux density $B$. With respect to the inner surface of the pipe wall and to the direction of pig motion one can define three components of $B$ : tangential $\left(B_{x}\right)$, transverse $\left(B_{y}\right)$, and normal $\left(B_{z}\right)$. Parameters such as a magnitude and spatial distribution of these components depend on a shape of the metal loss. Therefore, properly interpreted MFL signal provides information about geometry of the metal loss. In turn, this information can be used for evaluation of pipeline technical condition.

\section{CONSTRUCTION OF THE MFL PIG}

FLUMAG 500 MFL pig is a multi-module piston-like device. This pig measures magnetic flux leakage along an examined pipeline. It is designed for the inspection of a pipeline with wall thickness in the range of 5 to $20 \mathrm{~mm}$. The basic element of the pig is a magnetic circuit (Fig. 2) consisting of two magnets connected to each other by a magnetic keeper, and a set of Hall sensors located between poles of the magnetic circuit. The set consists of ten units, each measuring three quantities: the component of magnetic flux density that is tangential to the direction of tool motion $\left(B_{x}\right)$, the component that is normal to the inner surface of the pipe wall $\left(B_{z}\right)$, and the gradient of $B_{z}$ in the direction of the tool motion $\left(\partial B_{z} / \partial x\right)$. A single unit scans an area up to several millimeters wide. By placing a series of the units side by side in the transverse direction, high spatial resolution on the whole circumference of a pipe is obtained by the pig.

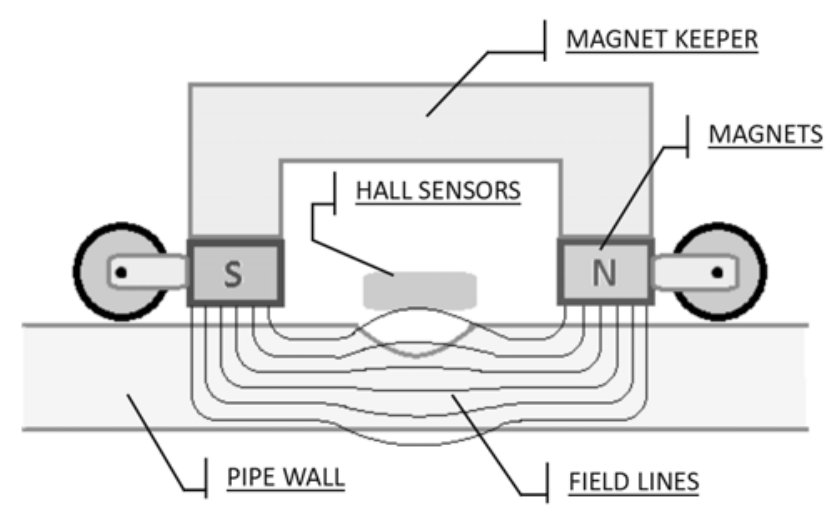

Fig. 2. Magnetic circuit

One can distinguish following components that make up FLUMAG 500 (Fig. 3):

- a battery unit containing lithium-ion cells that supply embedded electronics of the pig during inspection run;

- two measurement modules with sets of the magnetic circuits that magnetize the pipe wall and measure magnetic flux leakage;

- an odometer recording a distance traveled inside an examined pipeline;

- the inertial measurement unit consisting of three perpendicularly positioned accelerometers and fiber optic gyroscopes;

- a set of cups sealing the pig to the inner surface of a pipe wall, and thus allowing the pig to move with an extruded medium; 
- the extremely low frequency transmitter; the antenna signal can be detected outside a pipeline, which allows to determine a pig location in case of its malfunction or stuck; the transmitter is an independent module with its own power source.

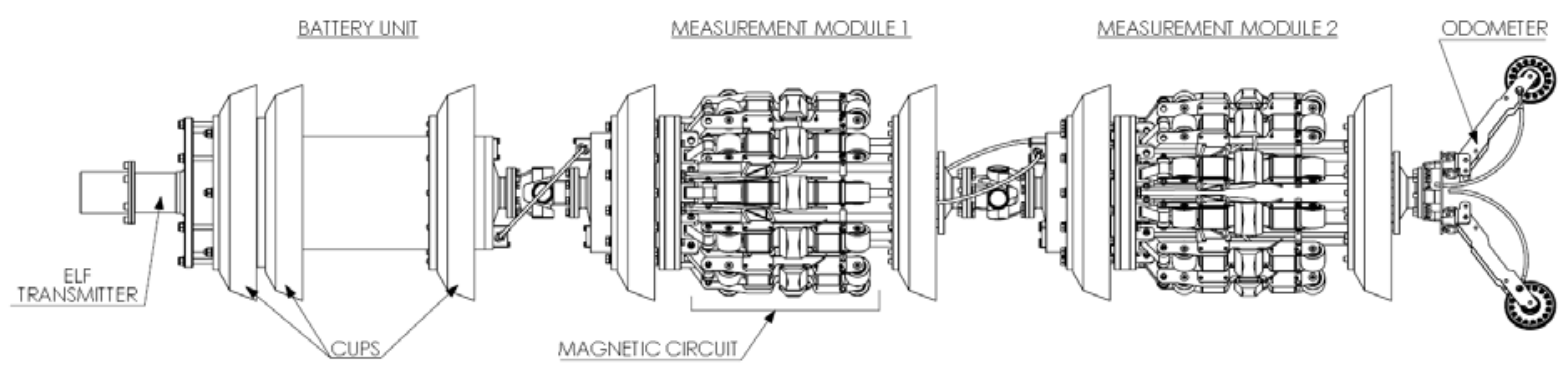

Fig. 3. Construction of FLUMAG 500

CDRiA Ltd. has a big experience in the field of pipeline diagnostics but mostly in liquid media such as crude oil. Physical conditions in oil transmission pipelines are quite different from those in gas pipelines for which FLUMAG 500 was designed. Therefore, the research team had to meet a number of design challenges. One of them was a requirement to place both the magnetic circuit poles and the Hall sensors as close as possible to the wall surface. On the other hand, these elements need to be protected against a mechanical damage due to a contact with unevenness of the wall surface. Polyurethane was proposed as a protective material for the Hall sensors. Selected composition of polyurethane has an excellent resistance to abrasion, stretching, tearing, as well as to many aggressive environments including solvents, acids, and petroleum products. In addition to mechanical and chemical protection of the sensors, the key issues of the pig design were: integrity of the capsules carrying on-board electronics and their resistance to pressure of an extruded medium, the right choice of a power source that allows continuous operation of the pig inside a pipeline, even on the distance of $200 \mathrm{~km}$.

\section{EXPERIMENTAL AND ANALYSIS METHODS}

The most time-consuming and demanding of an innovative approach was the analysis and interpretation of measured data. Whole this process can be divided into three stages. The first stage comprises preliminary preparation of data and signal processing. This is required due to signal distortions caused by factors such as a system digital noise, velocity-induced eddy current [5], changes of sensor lift-off and orientation during the inspection. Various techniques designed for signal and image processing can be used in this case, e.g. digital filtering, adaptive filtering [6], wavelet analysis [7], etc. The second step consists of detection and classification of signal features that are associated with defects in a pipe wall. At this stage it is crucial to distinguish signal features associated with defects from those associated with other factors, e.g. other material discontinuities (particularly weld zones and heat affected zones), and metal objects in vicinity of a pipe. The third stage consists in quantitative analysis of the signal i.e. determination of dimensions of detected defects. Wavelet transform can be used at this stage [7]. However, classification models based on machine learning methods, such as artificial neural networks, are often used for this purpose as well [8]. Network training is usually performed using data obtained for a set of artificial defects with 
known geometry. The main task of the model is to estimate dimensions of an identified defect, i.e. length, width, depth, internal or external location. This estimation is burdened with additional uncertainty due to velocity-induced eddy current that occurs in vicinity of a defect. This eddy current can significantly modify baselines of MFL signal components as well as amplitudes of peaks that are related to defects. The modification of the baselines consists in their offset that is proportional to the MFL tool velocity. This offset can be compensated with the use of information about temporary velocity of the tool that is provided by an odometer [9]. Compensation of an MFL signal amplitude is more difficult task because there is no general relation between amplitudes of the MFL signal components and the MFL tool velocity [9]. Some attempts were made to compensate the velocity effect on the amplitudes using data obtained by finite element method [5,10-12]. However, none of them utilizes experimental data that provide more reliable results of compensation.

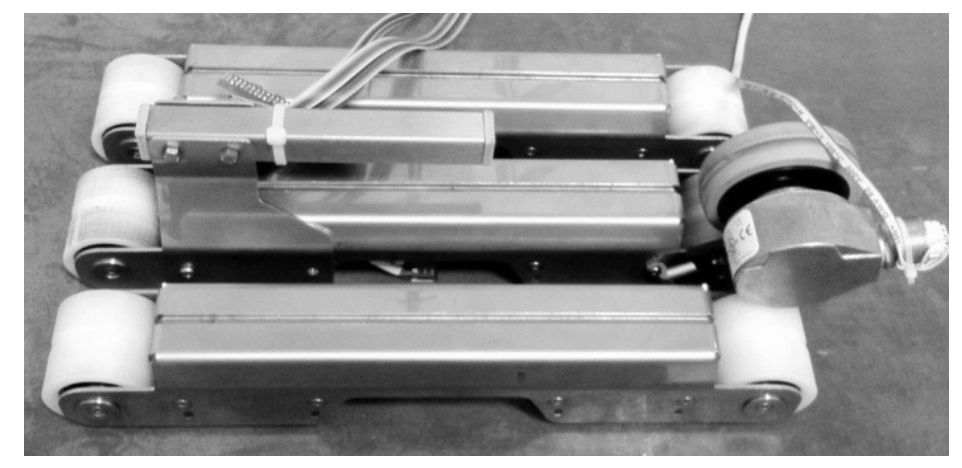

Fig. 4. Assembly of three magnetic circuits used at the development stage

At the development stage a number of samples containing artificial defects were examined. At this stage the MFL tool consisted of an odometer and three magnetic circuits connected to each other (Fig. 4). The samples had the form of plates made of 18G2A steel. The artificial defects had different dimensions and shapes. Some defects had a simple, rectangular geometry and differ only in one dimension as in the case of the test plate ' $\mathrm{N}$ ' described in Fig. 5. Thickness of the test plate ' $\mathrm{N}$ ' is equal to $10 \mathrm{~mm}$. Other defects were intended to imitate metal corrosion pits and were milled on the surface of the test plate 'I'. Their dimensions are presented in Fig. 6. As a result, the MFL signal dependence on dimensions and shape of a defect was investigated.

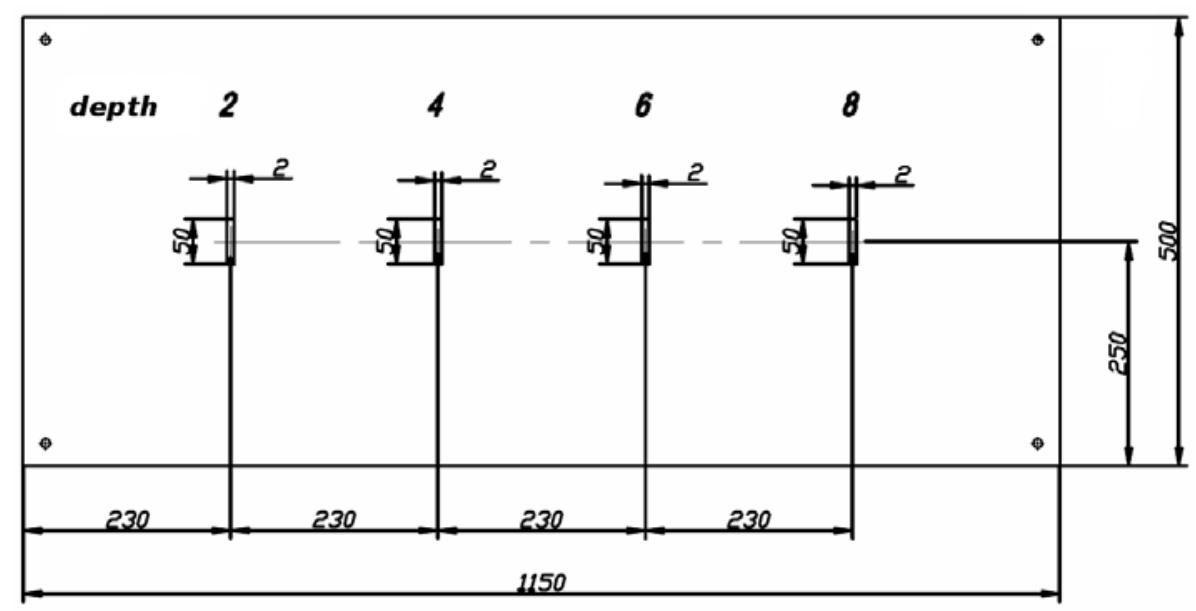

Fig. 5. Rectangular defects with different depths on the surface of the test plate ' $N$ ' 

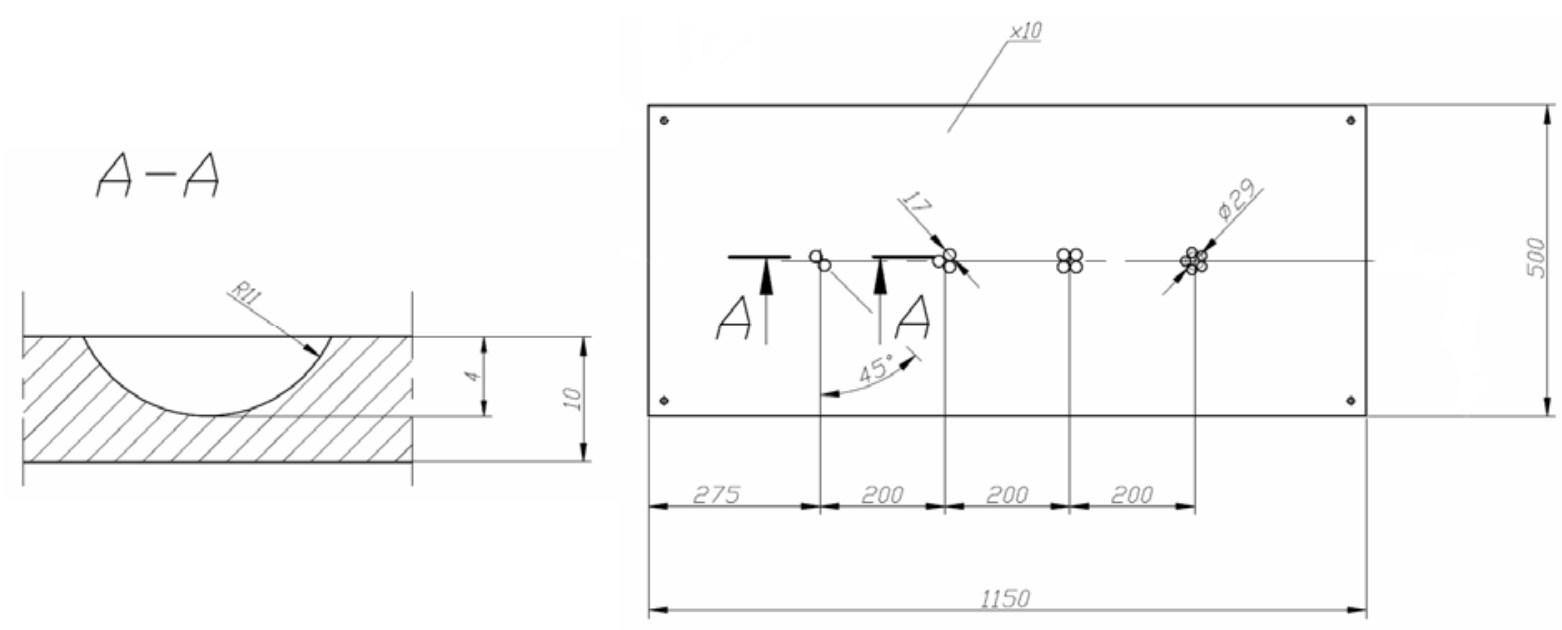

Fig. 6. Artificial defects imitating corrosion pits on the surface of the test plate 'l'

\section{RESULTS AND DISCUSSION}

Fig. 7 shows the results obtained for the test plate ' $N$ '. These results clearly demonstrate that the components of the MFL signal are subjected to significant changes in a region of a defect. For a rectangular slot with a relatively short length the characteristic form of the MFL signal is recorded. In this case, $B_{x}$ has the form of a positive peak. The waveform of $B_{z}$ is uneven and it has two peaks. $\partial B_{z} / \partial x$ as a derivative of $B_{z}$ is characterized by one major negative peak and two minor positive peaks. Amplitudes of the signal components are strongly dependent on the defect depth. Correlation between the amplitudes of the signal components and the defect depth enables to formulate a calibration function, which can be used for solving an inverse problem in a case of unknown geometry of a defect.

Signals recorded for the test plate 'I' are presented in Fig. 8. Four groups of artificial corrosion pits were milled on the surface of the plate. Each consisted of different number of cavities which stay in mutual contact by their edges. As a result, a waveform of each measured signal component is a bit more complex for this plate as compared to the signal measured for the test plate ' $\mathrm{N}$ ', because it is a superposition of flux leakages coming from adjacent cavities. Although the bowl-like cross section of these cavities are different from the rectangular cross section, in both cases waveforms of each components are characterized by the same features i.e. a kind of symmetry, number of peaks, etc. Also in the case of the test plate 'I', an amplitude of a selected waveform is close correlated to a defect maximal depth which is equal to $4 \mathrm{~mm}$ for all the defects.

Results obtained for the presented two test plates as well as for a number of other plates were used as training data for a machine learning process which led to the model enabling for the quantitative analysis of defects. 


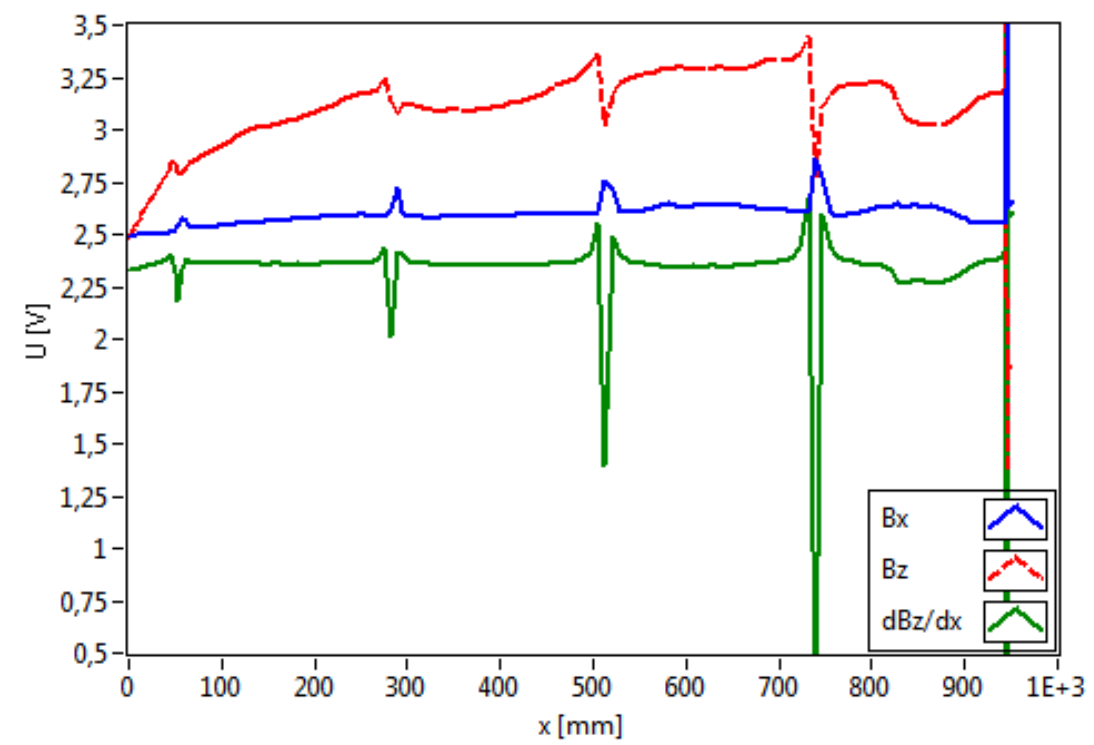

Fig. 7. The MFL signal obtained for the test plate ' $N$ '

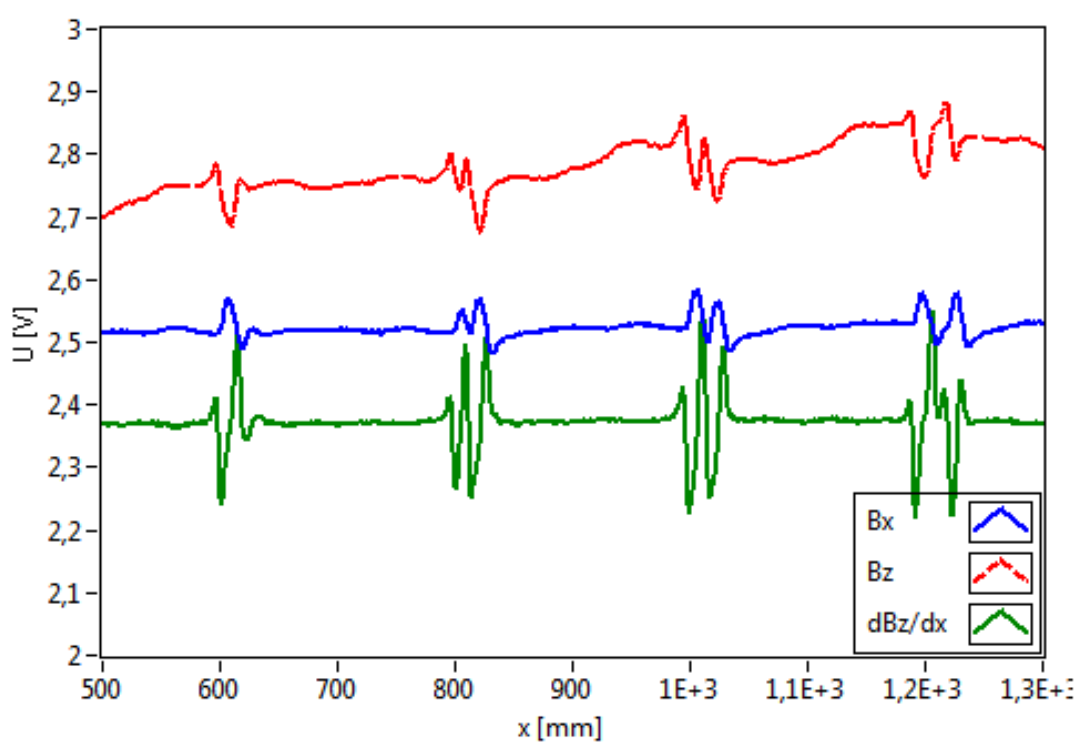

Fig. 8. The MFL signal obtained for the test plate 'l'

Finally, FLUMAG 500 was used to examine the technical condition of a working gas pipeline with a diameter of 20 " and the nominal wall thickness of $9 \mathrm{~mm}$. Obtained data were quantitatively analyzed using a model trained by artificial neural networks algorithms. The aim of the quantitative analysis was estimation of dimensions of detected metal losses. Accuracy of the estimation increases proportionally to a depth of a metal loss. It is a consequence of a higher signal-to-noise ratio as well as less significant velocity effect in a case of a relatively deep metal loss [9]. As an example of the analysis results, a portion of the signal measured for the pipeline is shown in Fig. 9. A metal loss located at the distance of $0.5 \mathrm{~m}$ is classified as a circumferential groove that is $10 \mathrm{~mm}$ long and $27 \mathrm{~mm}$ wide. Its maximal depth is estimated at $29 \%$ of the wall thickness. 


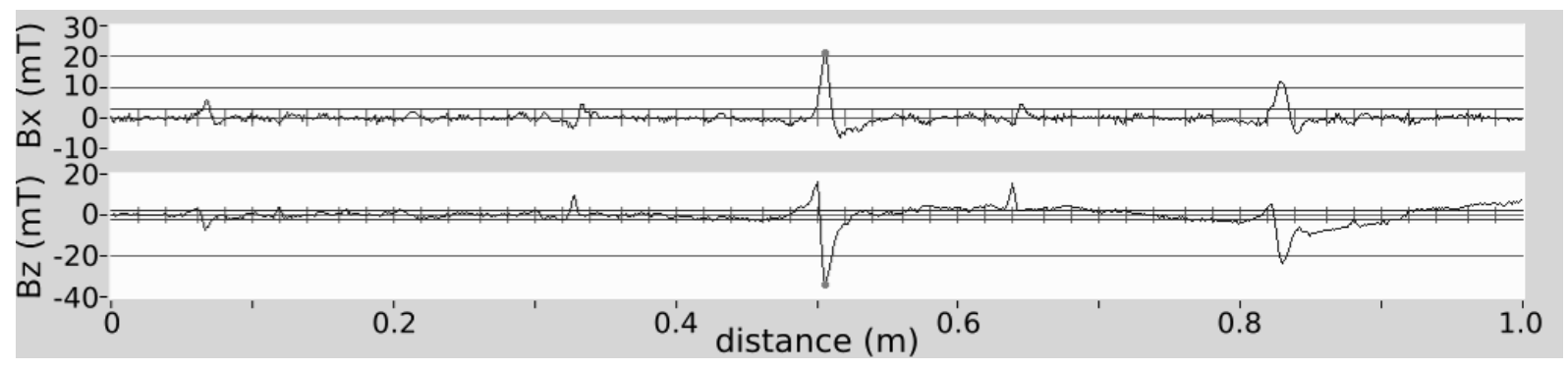

Fig. 9. A portion of the signal measured by FLUMAG 500 during the inspection of a working pipeline

\section{CONCLUSION}

The results of preliminary tests performed on the test plate 'I' showed that the prototype of the MFL tool enabled to locate and distinguish adjacent defects which had a shape and a depth very similar to real corrosion pits. These results as well those obtained for the other test plates were used for the training and the evaluation of the model which enabled estimation of defect dimensions. The final prototype of the magnetic circuit was adopted as a main functional part of the MFL pig called FLUMAG 500. With the use of FLUMAG 500 a working natural gas pipeline was investigated. Estimations of defects dimensions made for the signal measured during the inspection of the working pipeline, although not validated by another method, are physically correct and consistent with the experience of the CDRiA crew.

Accurate reproduction of corrosion defect dimensions, especially its depth, is crucial in the context of the remaining strength prediction of a pipeline. A pipeline inspection performed by a pig should give an answer for the question of whether a pipeline can safely continue its operation. Evaluation of the pipeline safety is often based on determination of maximum allowable operating pressure [13]. A method proposed by the American Society of Mechanical Engineers requires acquaintance of pipeline material characteristics, i.e. pipe nominal diameter and thickness, information about known metal losses, especially their maximum depth and length. FLUMAG 500 provides information not only about locations of metal losses, mainly corrosion pits, but also about their estimated dimensions. Therefore, it is suitable tool for evaluation of the gas pipeline safety.

\section{ACKNOWLEDGEMENTS}

This work was supported by the National Centre for Research and Development, Poland [project INNOTECH-K2/IN2/53/182767/NCBR/12].

\section{REFERENCES}

1. Ahmad, Z., Principles of corrosion engineering and corrosion control. Butterworth-Heinemann, 2006.

2. Porter P.C., Use of magnetic flux leakage (MFL) for the inspection of pipelines and storage tanks. Proc. SPIE 2454, Nondestructive Evaluation of Aging Utilities (1995), 172-184. 
3. Bubenik T.A., Nestleroth J.B., Eiber R.J., and Saffell B.F., Magnetic flux leakage (MFL) technology for natural gas pipeline inspection. Topical report, November 1992.

4. Sutherland J. and Paz H., Advances in in-line inspection technology for pipeline integrity. 5th Annual International Pipeline Congress, Morelia, Mexico, 2000.

5. Park G.S., Park S.H., Analysis of the velocity-induced eddy current in MFL type NDT. IEEE Trans. Magn. 40 (2004), 663-666.

6. Afzal M., Udpa S., Advanced signal processing of magnetic flux leakage data obtained from seamless gas pipeline. NDT\&E Int. 35 (2002), 449-457.

7. Piotrowski L., Chmielewski M., Analysis of the magnetic flux leakage signal detected by a pipeline inspection gauge with the help of the continuous wavelet transform. Journal of Electrical Engineering 66 (2015), 182-185.

8. Carvalho A.A., Rebello J.M.A., Sagrilo L.V.S., Camerini C.S., and Miranda I.V.J., MFL signals and artificial neural networks applied to detection and classification of pipe weld defects. NDT\&E Int. 36 (2006), 661-667.

9. Usarek Z., Influence of sample geometry, magnetic properties and a method of magnetisation on the spatial distribution of the stray magnetic field.. $\mathrm{PhD}$ dissertation (in Polish), Gdańsk University of Technology, 2017.

10. Mandayam S., Udpa L., Udpa S.S., and Lord W., Signal processing for in-line inspection of gas transmission pipelines. Res. Nondestruct. Eval. 8 (1996), 233-247.

11. Lei L., Wang C., Ji F., and Wang Q., RBF-based compensation of velocity effects on MFL signals. Insight 51 (2009), 508-511.

12. Lu S., Feng J., Li F., and Liu J., Precise Inversion for the Reconstruction of Arbitrary Defect Profiles Considering Velocity Effect in Magnetic Flux Leakage Testing. IEEE Trans. Magn. 53 (2017), 1-12.

13. Manual for Determining the Remaining Strength of Corroded Pipelines, Supplement to ASME B31 Code for Pressure Piping. An American National Standard - ASME B31G-2012. 